\title{
Malignant lymphoma of the testis: a study of 12 cases
}

\author{
Andrea G. Lantz, MD; ${ }^{*}$ Nicholas Power, MD; ${ }^{\dagger}$ Brian Hutton, $M S c{ }^{\S}{ }^{\S}$ Rekha Gupta, MD ${ }^{* *}$
}

\section{Abstract}

Introduction: Testicular lymphoma is a rare and deadly disease representing $1 \%$ to $2 \%$ of all non-Hodgkin lymphomas (NHLs) and approximately $5 \%$ of all testicular neoplasms. Our objective is this study was to identify the presenting signs and symptoms, treatment and outcome of patients with testicular lymphoma diagnosed at our institution from 1992 to 2005, and to identify any differences in survival based on Ann Arbor Stage and International Prognostic Index (IPI).

Methods: A retrospective chart review was performed to identify demographic characteristics, presenting signs and symptoms, treatment and outcomes. Survival was assessed using Kaplan-Meier survival curves and log-rank testing.

Results: Thirteen cases were identified; 1 of these cases was ultimately excluded due to a diagnosis of lymphoma a year before he presented with testicular involvement. Of the remaining 12 cases, the mean age was 65 years. Most patients presented with testicular and scrotal swelling or mass. B symptoms (weight loss, fever, night sweats) were present in 1 case. Of the 12 cases, 7 cases were stage I, 1 stage II and 4 stage IV. International Prognostic Index was low risk $(\leq 1)$ in 7 cases and high risk $(>1)$ in 4 cases. Orchiectomy was performed in all cases. Three patients received no further treatment. Of the remaining 9 patients, overall 7 received systemic chemotherapy, and 7 radiation therapy (prophylactic in 6). Three patients received intrathecal chemotheraphy prophylaxis. Seven patients achieved complete remission. Four patients (57\%) relapsed following complete remission. Median time to relapse was 32 months (range 11 to 73 months). Six patients died. Median survival was 29 months, and was significantly different between early versus advanced stage (stage I/II disease: 71 months; stage IV: 5 months $p=0.007$ ).

Conclusion: Testicular lymphoma is a rare and deadly form of extranodal lymphoma. Survival was significantly different in early stage I/II and IPI low-risk versus advanced stage IV and IPI high-risk disease. Randomized, prospective treatment trials may help to establish better treatment strategies.

Can Urol Assoc J 2009;3(5):393-8

\section{Résumé}

Introduction : Le lymphome testiculaire est une maladie rare et meurtrière représentant de 1 à $2 \%$ de tous les lymphomes non hodgkiniens ( $\mathrm{LNH}$ ) et environ $5 \%$ des cancers testiculaires. L'objectif de notre étude était de dégager les signes et symptômes initiaux, le traitement et les résultats possibles liés au lymphome testiculaire chez les patients ayant reçu un tel diagnostic à notre établissement entre 1992 et 2005, et de déterminer les différences dans les taux de survie en fonction de la classification de Ann Arbor et de I'Indice pronostique international (IPI).

Méthodologie : Un examen rétrospectif de dossiers a été effectué afin de dégager les caractéristiques démographiques, les signes et symptômes initiaux, les méthodes de traitement et les résultats. La survie a été évaluée à l'aide des courbes de survie de Kaplan-Meier et de la méthode de Mantel-Haenzel.

Résultats : Treize cas ont été cernés; un des cas a plus tard été exclus de notre étude en raison d'un diagnostic de lymphome établi un an avant la découverte de l'atteinte testiculaire. Chez les 12 cas restants, I'âge moyen était de 65 ans. La plupart des patients présentaient une enflure ou une masse testiculaires ou scrotales. Des symptômes B (perte de poids, fièvre, sueurs nocturnes) ont été notés dans un cas. Sur les 12 cas, 7 étaient de stade I, 1 , de stade II et 4 , de stade IV. L'IPI indiquait un faible risque $(\leq 1)$ dans 7 cas, et un risque élevé ( $>1)$ dans 4 cas. Tous les patients ont subi une orchidectomie. Trois patients n'ont reçu aucun autre traitement, tandis que chez les 9 autres patients, 7 ont reçu une chimiothérapie systémique et 7 , une radiothérapie (prophylactique dans 6 cas). Trois patients ont reçu un traitement prophylactique par chimiothérapie intrathécale. Sept patients ont obtenu une rémission complète, parmi lesquels quatre patients (57\%) ont présenté plus tard une récidive. Le délai médian avant la récidive était de 32 mois (écart : 11 à 73 mois). Six patients sont décédés. La survie médiane était de 29 mois; une différence significative a été notée sur le plan de la survie médiane entre les patients de stade précoce et les patients de stade avancé (stades I ou II : 71 mois; stade IV : 5 mois; $p=0,007$ ).

Conclusion : Le lymphome testiculaire est une forme rare et meurtrière de lymphome extraganglionnaire. La survie s'est révélée significativement différente entre les stades précoces (I/II) et un IPI associé à un faible risque d'une part et un stade avancé (IV) et un IPI associé à un risque élevé d'autre part. Des essais prospectifs avec répartition aléatoire pourraient aider à élaborer de meilleures stratégies thérapeutiques.

\section{Introduction}

Testicular lymphoma is a rare and deadly disease representing $1 \%$ to $2 \%$ of all non-Hodgkin lymphomas (NHLs) and approximately $5 \%$ of all testicular neoplasms. ${ }^{1-3}$ It is predominantly a disease of the elderly ${ }^{3}$ and is the most common testicular tumour in men over the age of $60 .{ }^{4}$ The 
usual presentation is a painless unilateral testicular mass or swelling, ${ }^{4-7}$ with bilateral disease present in some cases. The most common form of testicular lymphoma is diffuse large B-cell lymphoma (DLBCL). 2,6,7 Secondary involvement of the testis by NHL is more common than primary extranodal disease. ${ }^{4}$

Due to the small number of cases of primary testicular lymphoma, there are few prospective treatment trials. ${ }^{2,8}$ Retrospective studies report treatment with orchiectomy, various forms of chemotherapy and/or radiation, ${ }^{3,6,7,9-14}$ with some cases including prophylactic radiation and/or intrathecal chemotherapy. Testicular lymphoma shows a propensity towards systemic dissemination and relapse even years after complete response (CR) to treatment. ${ }^{1,14,15}$ Extranodal and central nervous system (CNS) relapse is common. ${ }^{15,16}$

The goal of this study was to review the cases of testicular lymphoma diagnosed at our institution to add to the current knowledge of the presentation, workup, treatment and outcome of this disease, and to determine if there was a significant difference in survival by stage and International Prognostic Index (IPI) score.

\section{Methods}

Patients with testicular lymphoma diagnosed at the Queen Elizabeth II Health Sciences Centre in Halifax, Nova Scotia, Canada, were included in this retrospective cohort study. Cases diagnosed between July 1992 and September 2005 were identified by performing a search of the pathology SNOMED (Systematized Nomenclature of MEDicine - Clinical

\begin{tabular}{ll}
\hline Table 1. Ann Arbor Staging System \\
\hline Stage & Description \\
\hline I & $\begin{array}{l}\text { Involving a single lymph node region (I) or single } \\
\text { extralymphatic organ or site (IE) }\end{array}$ \\
\hline II & $\begin{array}{l}\text { Two or more involved lymph node regions on the } \\
\text { same side of the diaphragm (II) or localized } \\
\text { involvement of an extralymphatic organ or site (IIE) }\end{array}$ \\
\hline III & $\begin{array}{l}\text { Lymph node involvement on both sides of the } \\
\text { diaphragm (III), or localized involvement of an } \\
\text { extralymphatic organ or site (stage IIIE), or spleen } \\
\text { (IIIS), or both (IIIES) }\end{array}$ \\
\hline IV & $\begin{array}{l}\text { The presence of diffuse or disseminated involvement } \\
\text { of one or more extralymphatic organs (e.g., liver, } \\
\text { bone, marrow, lung), with or without associated } \\
\text { lymph node involvement }\end{array}$ \\
\hline
\end{tabular}

The presence or absence of systemic symptoms should be noted with each designation: A for asymptomatic, B for presence of fever, sweats or weight loss $>10 \%$ of body weight.

Taken from Moormeier et al. ${ }^{17}$
Terms) database. All available files were reviewed. Demographic information, presenting signs and symptoms, investigations, stage of disease, treatment and outcome including response rate, relapse site, relapse treatment and overall survival were recorded.

Staging was performed according to the Ann Arbor Staging system (Table 1). ${ }^{17}$ Cases were classified according to a modified version of the IPI (Table 2). ${ }^{18}$

Survival was assessed by constructing Kaplan-Meier survival curves and performing log-rank testing. For the purpose of our study, we defined CR as absence of disease for a minimum of 1 month following treatment. Partial response (PR) was defined as $>50 \%$ reduction in disease burden or progression of disease with treatment. Overall survival (OS) and progression-free survival (PFS) were calculated from the date of diagnosis to death and progression or date of last follow-up. In analyses of PFS, patients who died without experiencing disease progression were censored at the time of death.

\section{Results}

\section{Patient characteristics}

Between July 1992 and September 2005, 13 patients were diagnosed as having testicular lymphoma. One patient was excluded from the study due to a diagnosis of lymphoma 1 year prior to presenting with testicular involvement. The remaining 12 patients had no prior diagnosis of hematologic malignancy. The mean age at diagnosis was 65 , with a median of 70 (range 19 to 84).

The most common presenting symptom was unilateral testicular/scrotal swelling or mass (9 out of 12), which was associated with pain or ache in 1 case (patient \#3). One patient presented with bilateral swelling $(\# 11)$. One patient presented with hypercalcemia, dizziness and confusion (\#11) and the remaining patient (\#12) presented with painless jaundice secondary to an abdominal mass. In both of these patients (\#11 and \#12), unilateral testicular masses were found on physical examination. B symptoms (fever, sweats, and/or

\begin{tabular}{lcc}
\hline \multicolumn{4}{l}{ Table 2. International Prognostic Index } \\
\hline Characteristic & 0 & 1 \\
\hline Age, yrs & 60 & $>60$ \\
\hline Serum LDH & $1 \times$ normal & 1 \\
\hline Performance status & 0 or 1 & $2-4$ \\
\hline Stage & I or II & III or IV \\
\hline $\begin{array}{l}\text { Taken from the International Non-Hodgkin's Lymphoma Prognostic Factors Project. } \\
\text { LDH }=\text { lactate dehydrogenase. }\end{array}$
\end{tabular}


significant weight loss) were present in 1 case (\#12).

Staging was performed with thoracic, abdominal and pelvic computed tomography scan in all patients, bone marrow biopsy in 10 patients, and Gallium scan in 9 patients. Over half of all patients (7 of 12) had stage I disease, 1 had stage II disease and 4 had stage IV disease. The lactate dehydrogenase (LD) level was elevated in 3 patients (1 stage I, 2 stage IV). According to the IPI score, 7 patients had low-risk disease (IPI $\leq 1)$ and 5 had high-risk disease $(\mathrm{IPI}>1)$. IPI score was very similar to Ann Arbor Stage with only 1 stage I patient being high-risk.

\section{Pathologic features}

According to the World Health Organization classification system, all were B-cell lymphomas: 11 of the 12 cases were diffuse large B-cell lymphoma (DLBCL), 1 of which was anaplastic. The remaining case was lymphoblastic lymphoma.

\section{Radiographic features}

Ultrasound findings were consistent with either focal or diffusely decreased echogenicity within the testicle. Doppler ultrasound showed diffuse increasedflow within the mass. Most of the affected testicles were enlarged.

\section{Treatment}

Table 3 provides a synopsis of the clinical information, treatment and outcomes. All patients underwent orchiectomy, which was bilateral in 1 case. Three had orchiectomy alone (1 patient died before further treatment was possible [\#11], and 2 patients declined further treatment $[\# 9,10])$. Additional information was unavailable for one patient (\#4). One patient

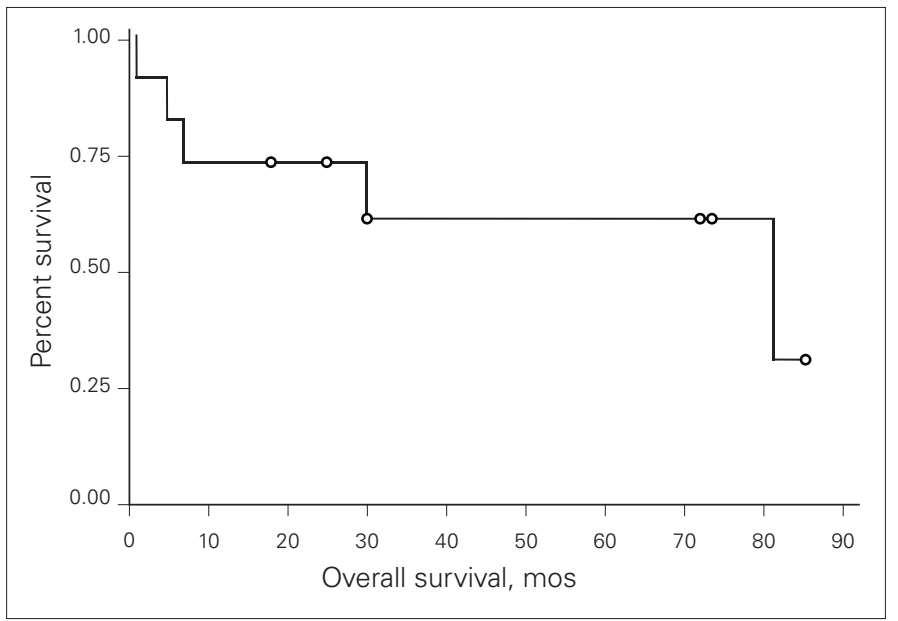

Fig. 1. Overall survival in patients in the studied group of 11 patients. received orchiectomy and chemotherapy. One patient had orchiectomy and radiation. Six patients had orchiectomy, chemotherapy and radiation. Overall, 7 patients received chemotherapy (64\%). Five received CHOP (cyclophosphamide, doxorubicin, vincristine) and prednisone, 1 had VACOP-B (etoposide, doxorubicin, vincristine, prednisone, bleomycin) and 1 patient with lymphoblastic lymphoma had the Coleman protocol. Prophylaxis with intrathecal methotrexate was given in 3 cases. Radiation was given to 7 patients overall. Six (54\%) were given prophylactic radiation: 1 cranial, 3 to scrotum/contralateral testis and 2 to scrotum/contralateral testis and ipsilateral pelvic/paraaortic lymph nodes.

\section{Outcomes}

Two patients who declined treatment after orchiectomy had partial remission with disease progression at 4 months (death) and 8 months (cutaneous relapse) after diagnosis. Two patients had disease progression prior to or during treatment. The remaining 7 patients $(64 \%)$ had complete remission. Four patients $(57 \%)$ relapsed following CR. Median time to relapse was 32 months (range 11 to 73 months). Patients with relapse following CR showed relapse involving the CNS $(n=2)$, skin $(n=4)$, head and neck $(n=1)$, and abdominal/retroperitoneal lymph nodes $(n=3)$ and pleural effusion $(n=1)$. There were no isolated nodal relapses.

\section{Overall and progression-free survival}

Out of the 11 patients for whom data was available, 6 died and 5 lived. The median survival for all patients was 29 months (Fig. 1). By stage, for all patients included in the study, the median survival was 71 months (range 17 to 84 months) for

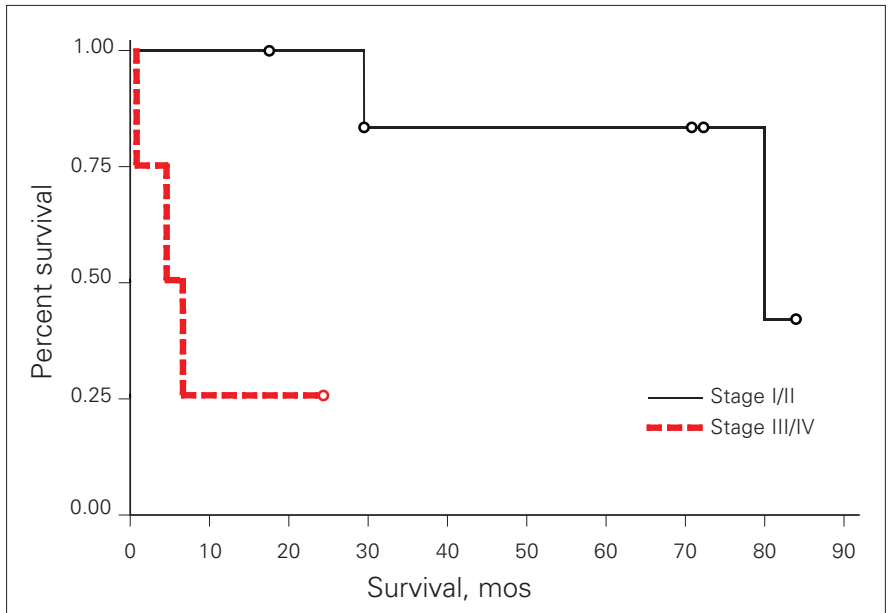

Fig. 2. Overall survival in patients with stage I/II versus III/IV. Log-rank: $p=0.007$. 


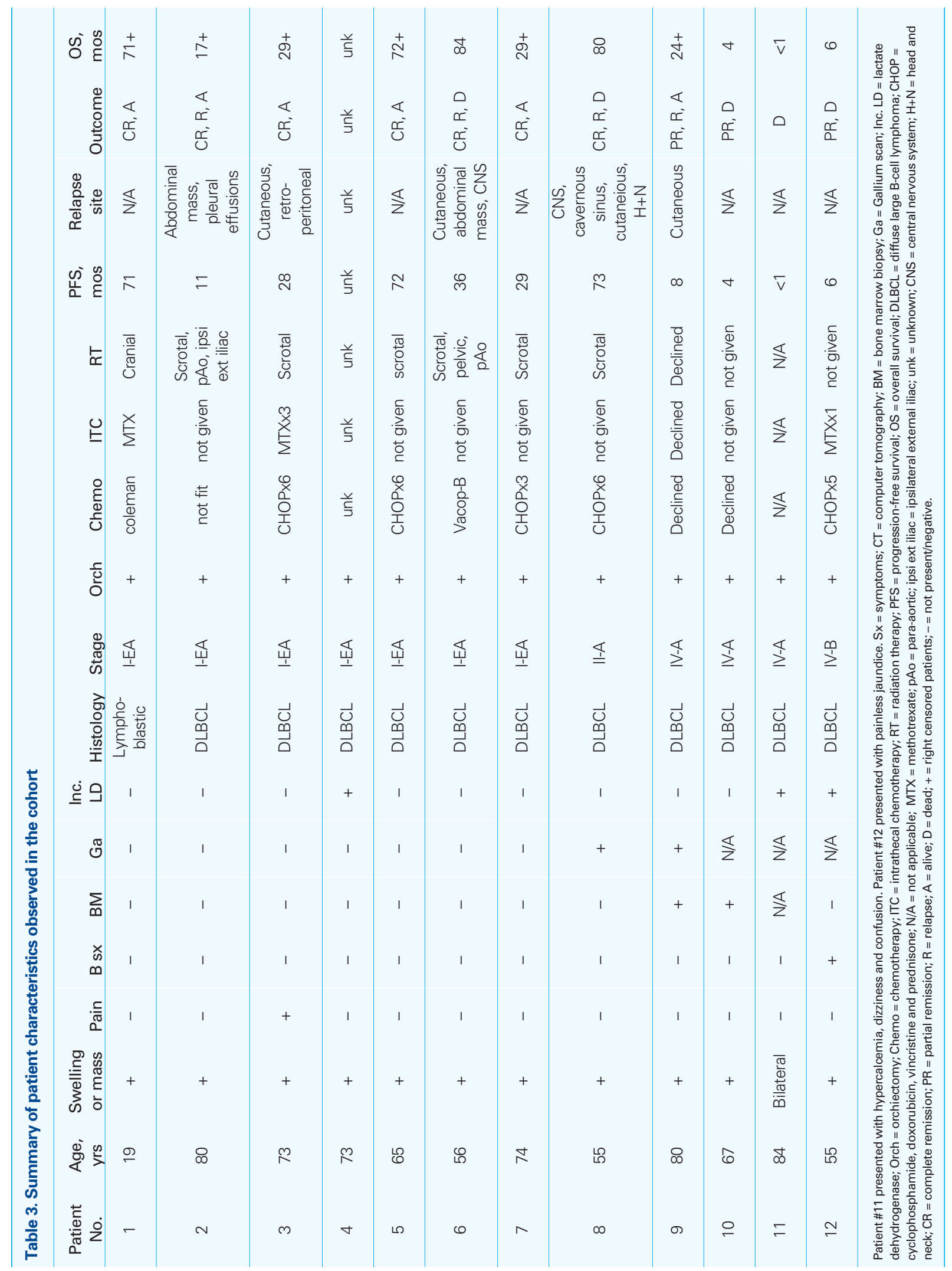




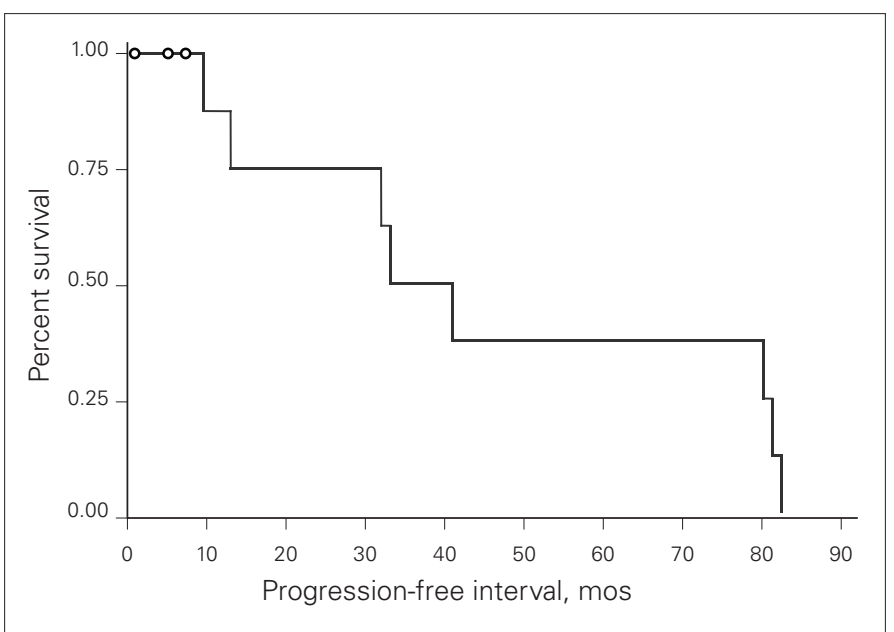

Fig. 3. Progression-free survival in the studied group of 11 patients.

stage I/II $(n=7)$ and 5 months for stage IV $(n=4)$ (Fig. 2). Log-rank testing identified a clinically significant survival difference between patients grouped by Ann Arbor Stage I/II versus IV for overall survival $(p=0.007)$. Ann Arbor Staging corresponded to IPI risk score, with almost all stage I/II patients being low-risk. Relapse or disease progression was seen in 8 patients (Fig. 3), with median time to relapse/progression of 36 months (range 11 to 73 months) in stage I/II and 5 months in stage IV disease $(p<0.001)$ (Fig. 4). Three patients did not experience relapse as of their most recent follow-up visits at 29, 71 and 72 months, respectively.

\section{Discussion}

Testicular lymphoma is a rare disease comprising approximately $1 \%$ to $2 \%$ of all NHLs and about $5 \%$ of testicular tumours, and is primarily a disease of the elderly. The median age in our study was 65 years (range 19 to 84 years), which is comparable to other reports. . $^{71,12,14,19}$ In our series, most patients presented with testicular swelling (10 unilateral, 1 bilateral, 2 painful), which is the most common presenting symptom for testicular lymphoma. ${ }^{4}$ One patient presented with obstructive jaundice from abdominal adenopathy. B symptoms were rare and present in only 1 case. Over half of patients had stage I disease, 1 had stage II disease and 4 had stage IV disease. The IPI score was low risk for all patients, but 1 stage I/II patient; the IPI score was high risk for all stage IV patients and 1 stage I patient.

Testicular lymphoma carries a poor prognosis compared to other NHL and extranodal lymphomas ${ }^{8,15}$ and may require a more prolonged course of chemotherapy compared to other extranodal lymphomas. ${ }^{20}$ Stage and pathologic grading are the most important predictive factors for outcome. ${ }^{21}$

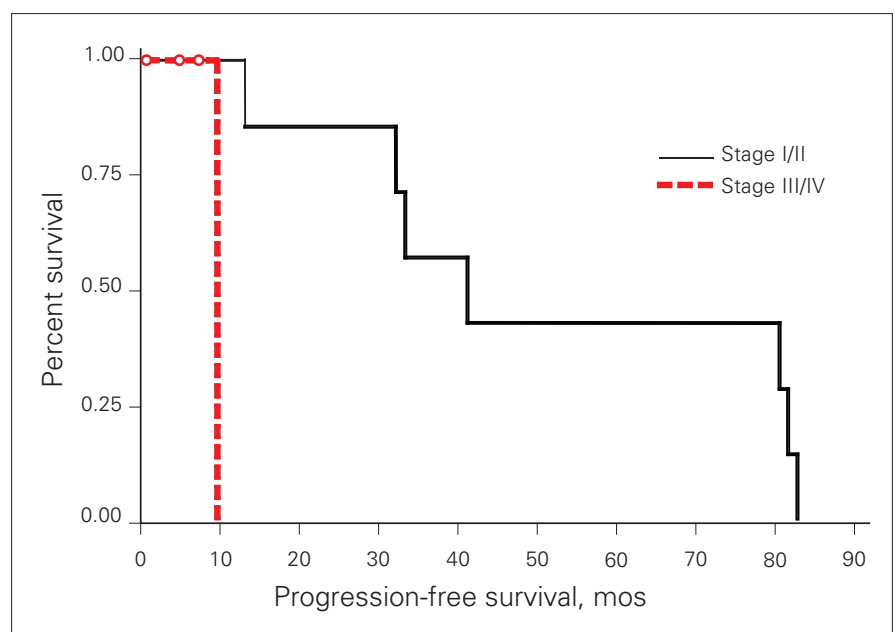

Fig. 4. Progression-free survival in patients with stage I/II versus III/IV. Logrank: $p=0.008$.

Younger age and early stage, which are part of the IPI, have been shown to be independent prognostic factors influencing the overall and disease-free survival. ${ }^{10}$

The first diagnostic and therapeutic procedure performed in testicular lymphoma cases is orchiectomy, ${ }^{11}$ which was performed in all cases in our series. Further optimal treatment is unclear because of the rarity of this disease, and because there are very few prospective studies ${ }^{2,8}$ and no randomized studies. Testicular lymphoma is a disease of the elderly, which may affect the choices of further therapies, such as multi-agent systemic chemotherapy. In our series, 3 patients did not receive chemotherapy because they were either unfit or declined further treatment due in part to their health status and poor prognosis. Treatment following orchiectomy included multi-agent chemotherapy in 7 cases (5 CHOP, 1 VACOP-B, 1 Coleman protocol). Seven patients received radiation, of which 6 were prophylatic. Three patients received CNS prophylaxis with intrathecal chemotherapy.

Due to the differences between early and advanced stages of testicular lymphoma, a complete lymphoma workup should be performed including bone marrow biopsy and CT scan of the abdomen and pelvis. Treatment regimens vary widely, as many studies on testicular lymphoma were done retrospectively, including our current series. ${ }^{4,9,15}$ Connors $^{22}$ advocates a phased-management approach for testicular lymphoma with all patients receiving multi-agent chemotherapy (doxorubicin, cyclophosphamide, vincrisitine and a corticosteroid). According to Connors, patients with early-stage disease IAE/IIAE should receive brief chemotherapy with involved field radiation including the contralateral testis; those with B symptoms, bulky nodal disease $(>10 \mathrm{~cm})$, or stage III/IV should receive more exten- 
sive chemotherapy with RT, and stage III/IV should also receive intrathecal chemotherapy. A more recent study advocates a more extensive course of chemotherapy in earlystage disease, ${ }^{20}$ which may not be feasible in an elderly patient. The treatment regimen advocated by Connors addresses 2 characteristics of this disease: the propensity to relapse in the opposite testicle and in the CNS. The risk of testicular relapse without prophylactic contralateral radiation is at least 25\%; this risk is essentially eliminated with radiation treatment. $3,15,16,19,22$ Most cases of testicular lymphoma are diffuse large B-cell, which are CD20+. ${ }^{2,6,7}$ The chimeric monoclonal anti-CD20 antibody, rituximab, has been shown to give improvement in progression-free and overall survival in cases of DLBCL. ${ }^{23}$ It is not known if the addition of rituximab would improve survival in cases of DLBCL of the testis.

In our series, the median survival for all patients was 29 months. Log-rank testing showed a significant difference in survival between early stage (I/II) versus advanced stage IV disease $(p=0.007)$. Median survival in stage I/II cases was 71 months compared to 5 months in stage IV. Time to disease progression or relapse was significantly different between stage I/II and IV cases, with median time to relapse/progression of 36 months ( 11 to 73 months) and 5 months, respectively $(p<0.001)$. Only 3 patients were well and relapse-free at latest follow-up of 21, 71 and 72 months. Our survival data are similar to those reported by other authors. $4,7,9,11,12,15$ Others have also found that patients with early-stage disease and low-risk IPI have a significantly longer disease-free and OS compared to patients with advanced stage and high-risk IPI score. ${ }^{11,15,19}$

\section{Conclusion}

Testicular lymphoma is a disease associated with a high incidence of recurrence and is associated with a poor prognosis. Early-stage disease or low-risk IPI tends to give a better survival compared to advanced stage or high-risk IPI. Even patients with early stage/low-risk disease are at high risk for recurrence years after initial diagnosis; therefore, these patients should have both short- and long-term follow-up. With the low incidence of testicular lymphoma and lack of prospective trials, collaboration and treatment within clinical trials including new agents, such as rituximab, may help to establish the best treatment regimen for this deadly disease.

From the *Department of Surgery, Division of Urology, University of Ottawa, OHtawa, ON; ${ }^{\dagger}$ Department of Urology, Dalhousie University, Halifax, NS; §Ottawa Health Research Institute, Ottawa, ON; ${ }^{\star *}$ Departments of Urology and Pathology, Dalhousie University, Halifax, NS
This paper has been peer-reviewed.

Competing interests: None declared.

\section{References}

1. Freeman C, Berg JW, Cutler SJ. Occurrence and prognosis of extranodal lymphomas. Cancer 1972;29: $252-60$.

2. Linassier C, Desablens B, Lefrancq T, et al. Stage H-IIE primary non-Hodgkin's lymphoma of the testis: Results of a prospective trial by the GOELAMS study group. Clin Lymphoma 2002;3(3):167-72.

3. Zucca E, Conconi A, Mughal TI, et al. Patterns of outcome and prognostic factors in primary large-cell lymphoma of the testis in a survey by the International Extranodal Lymphoma Study Group. J Clin Oncol 2003:21:20-7.

4. Shahab N, Doll DC. Testicular lymphoma. Semin Oncol 1999;26:259-69.

5. Zucca E, Roggero E, Bertoni F, et al. Primary extranodal non-Hodgkin's lymphomas. Part 1: Gastrointestinal, cutaneous and genitourinary lymphomas. Ann Oncol 1997;8:727-37.

6. Ferry JA, Harris NL, Young RH, et al. Malignant lymphoma of the testis, epididymis, and spermatic cord. A clinicopathologic study of 69 cases with immunophenotypic analysis. Am I Surg Pathol 1994;18: 376-90.

7. Darby S, Hancock BW. Localised non-Hodgkin lymphoma of the testis: The Sheffield Lymphoma Group experience. Int J Oncol 2005;26:1093-9.

8. Aviles A, Neri N, Huerta-Guzman J, et al. Testicular lymphoma: Organ-specific treatment did not improve outcome. Oncology 2004;67:211-4.

9. Lagrange JL, Ramaioli A, Theodore CH, et al. Non-Hodgkin's lymphoma of the testis: A retrospective study of 84 patients treated in the French anticancer centres. Ann Oncol 2001;12:1313-9.

10. Zouhair A, Weber D, Belkacemi Y, et al. Outcome and patterns of failure in testicular lymphoma: A multicenter rare cancer network study. Int J Radiat Oncol Biol Phys 2002;52:652-6.

11. Hasselblom S, Ridell B, Wedel H, et al. Testicular lymphoma — a retrospective, population-based, clinical and immunohistochemical study. Acta Oncol 2004;43:758-65.

12. Tondini C, Ferreri AJ, Siracusano L, et al. Diffuse large-cell lymphoma of the testis. J Clin Oncol 1999; 17: 2854-8.

13. Sasai K, Yamabe H, Tsutsui K, et al. Primary testicular non-Hodgkin's lymphoma: a clinical study and review of the literature. Am J Clin Oncol 1997;20:59-62.

14. Fonseca R, Habermann $T M$, Colgan JP, et al. Testicular lymphoma is associated with a high incidence of extranodal recurrence. Cancer 2000;88:154-61.

15. Touroutoglou N, Dimopoulos MA, Younes A, et al. Testicular lymphoma: late relapses and poor outcome despite doxorubicin-based therapy. J Clin Oncol 1995;13:1361-7.

16. Seymour JF, Solomon B, Wolf MM, et al. Primary large-cell non-Hodgkin's lymphoma of the testis: a retrospective analysis of patterns of failure and prognostic factors. Clin Lymphoma 2001;2:109-15.

17. Moormeier JA, Williams SF, Golomb HM. The staging of non-Hodgkin's lymphomas. Semin Oncol 1990;17:43-50.

18. A predictive model for aggressive non-Hodgkin's lymphoma. The International Non-Hodgkin's Lymphoma Prognostic Factors Project. N Engl J Med 1993;329:987-94.

19. Visco C, Medeiros $\downarrow$, Mesina OM, et al. Non-Hodgkin's lymphoma affecting the testis: is it curable with doxorubicin-based therapy? Clin Lymphoma 2001;2:40-6.

20. Shenkier TN, Voss N, Fairey R, et al. Brief chemotherapy and involved-region irradiation for limitedstage diffuse large-cell lymphoma: An 18-year experience from the British Columbia Cancer Agency. J Clin Oncol 2002;20:197-204.

21. Doll DC, Weiss RB. Malignant lymphoma of the testis. Am J Med 1986;81:515-24.

22. Connors JM. Problems in lymphoma management: special sites of presentation. Oncology (Williston Park) 1998;12:185,91; discussion 192-5.

23. Sehn LH, Donaldson J, Chhanabhai M, et al. Introduction of combined CHOP plus rituximab therapy dramatically improved outcome of diffuse large B-cell lymphoma in British Columbia. J Clin Oncol 2005;23:5027-33

Correspondence: Dr. Andrea G. Lantz, University of Ottawa, Department of Surgery, Division of Urology, Box 222, General Campus, The Ottawa Hospital, 501 Smyth Road, Ottawa, ON KIH 8L6; fax 613-761-5305; alantz@ottawahospital.on.ca 